\title{
Retrieving 3D medical data along fitted curved slices and their display
}

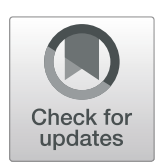

Marco Paluszny ${ }^{*} \mathbb{D}^{\dagger}$ and Dany Ríos ${ }^{\dagger}$

\begin{abstract}
Background: Computer tomography and magnetic resonance imaging are usually offered to the clinician in the form of sequences of axial, sagittal and coronal planar cuts. Frequently this does not allow for the full inspection of the morphology of the area of interest, because it is limited by the planarity. Efforts have been made to extract information along curved slices but their planar display is prone to metric deformation.

Methods: We propose a new visualization alternative of 3D medical volumes using curved slices adapted to areas of interest. We use surfaces fitted to specific organs as visualization canvasses. We describe the differential geometry techniques used to build the surfaces that may be isometrically flattened. These are referred to as develpable surfaces.

Results: We show concrete examples deemed useful for the development of clinical and educational tools. Our examples are centered in magnetic resonance data of the rotator cuff muscle complex and computed tomography data of maxillofacial and dental studies. We also look at the extraction and display of information from volumes of aortic aneurysms along transversal surfaces.

Discussion: We look at extensions of the technique and propose further possible clinical use of texturized surfaces in the context of volume navigation.

Conclusions: We presented a technique to extract information from computer tomography and magnetic resonance volumes, using two different texturization techniques. In the cases that the fitting surfaces are chosen to be developable, they may be flattened without distortion. We also discuss how tu use the technique in other visualization tasks such as volume navigation and detection of volumetric features.
\end{abstract}

Keywords: Computed tomography, Magnetic resonance, Developable surface, DICOM

\section{Background}

The adoption of imaging techniques to inspect $3 D$ body organs and clinically significant structures has been increasingly present in many medical and odontological areas. Already in 1989, the visionary work of Fuchs, Levoy and Pizer [1] points to the need of computer graphics techniques in medical imaging applications. The review of Zhang, Eagleson and Peters [2] sketches $2 D$ and $3 D$ visualization techniques in medicine. The recent work of Wheeler et al. [3] shows that it is feasible to build a $3 D$ interactive environments to inspect medical volumes, but it comes at a high computational cost. In fact, in the last twenty years the volume of $3 D$ medical data has been growing significantly [4], and this is being compounded with upward trends in archiving, sharing, and data availability. This provides a huge opportunity for the geometry community for development and testing of technologies for the visualization of medical volumes. One of the enabling factors is the existence of universally accepted dicom standard, which provides fairly structured reporting and allows for imaging study review with publicly available software ${ }^{1}$. In particular, Computed Tomography (CT) and Magnetic Resonance Imaging (MRI) are standard tools for internal organ visualization in dentistry, and in medical practice in general. But, if no sophisticated visualization software is available the CT or MRI

*Correspondence: marcopaluszny@gmail.com

${ }^{+}$Marco Paluszny and Dany Ríos contributed equally to this work.

Universidad Nacional de Colombia, Carrera 65, No. 59a, Medellín, Colombia

${ }^{1}$ Osirix DICOM viwer - http://www.osirix-viewer.com/resources/dicomimage-library/ for Mac OS and RadiAnt - https://idoimaging.com/programs/ 107, for Windows.

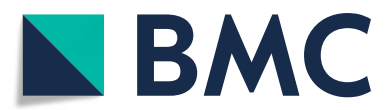

(c) The Author(s). 2020 Open Access This article is distributed under the terms of the Creative Commons Attribution 4.0 International License (http://creativecommons.org/licenses/by/4.0/), which permits unrestricted use, distribution, and reproduction in any medium, provided you give appropriate credit to the original author(s) and the source, provide a link to the Creative Commons license, and indicate if changes were made. The Creative Commons Public Domain Dedication waiver (http://creativecommons.org/publicdomain/zero/1.0/) applies to the data made available in this article, unless otherwise stated. 


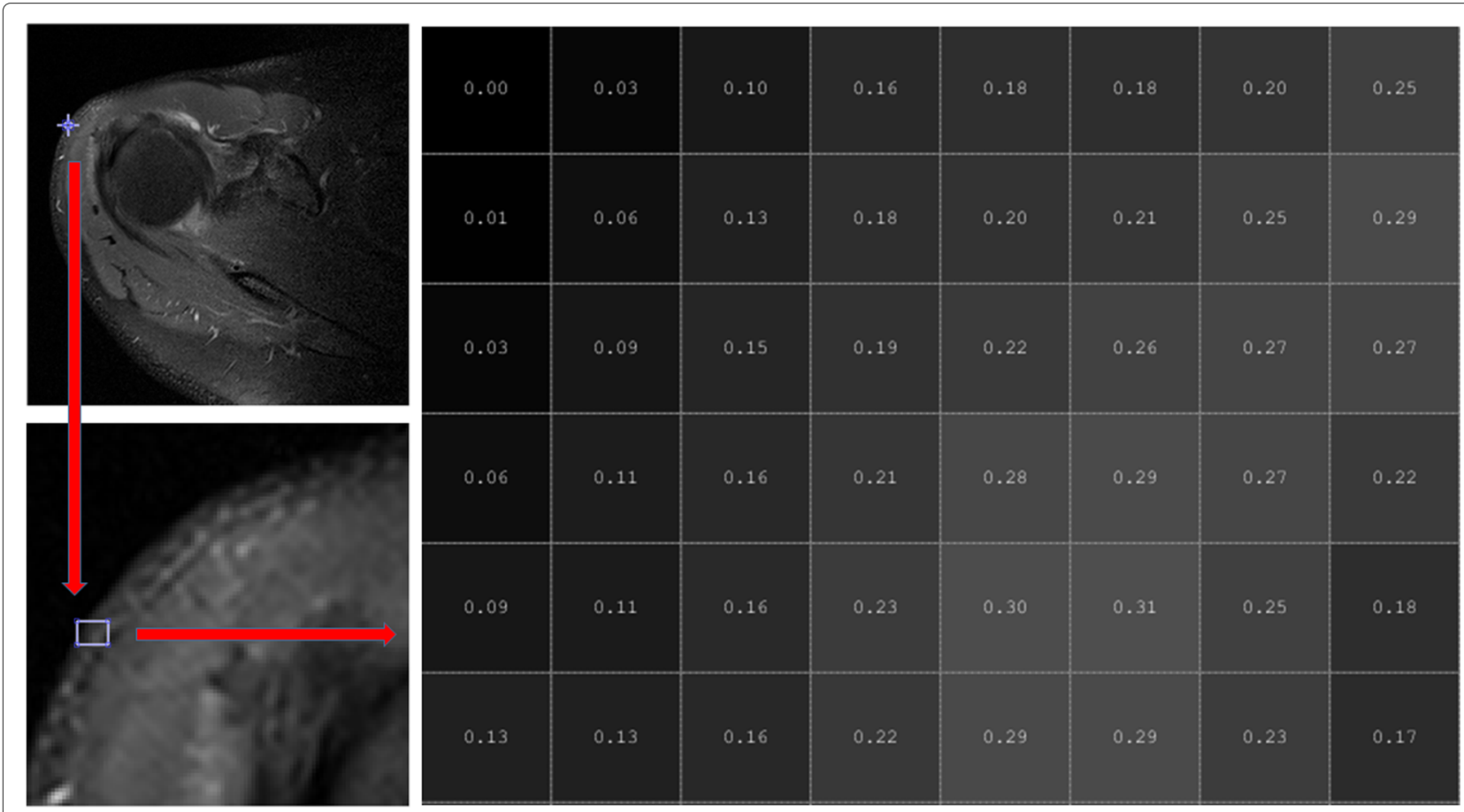

Fig. 1 Pixel values of a region of one slice of a rotator cuff MRI

information used for diagnosis is usually restricted to flat slices that can only be inspected sequentially ${ }^{2}$.

Any image to be inspected arises by setting each pixel to the gray level corresponding to the tissue type at its position in $3 D$, and this is referred to as the texturization process, see Fig. 1. The full set (usually ranging from under a hundred to a few hundred) of these planar images is referred to as a CT (or MRI) volume. Figure 2 shows some slices of a dental maxillofacial CT.

Usually the set of planar slices conforming a medical volume are parallel in $3 D$ and live in a rectangular box. Figure 3 illustrates the placement in $3 D$ of the planar slices of Fig. 2. Note that the slices do not have to be equidistant in space. For some organs, it may happen, that the actual positions of the slices in $3 D$ space are not parallel in $3 D$. For, an example of six stomach slices and their actual positions in space is illustrated in Figs. 4 and 5.

\section{Methods}

DICOM (Digital Imaging and Communications in Medicine) is a communications protocol and file format to store medical information from imaging studies. Each .dcm file contains the gray levels of one slice of a body part. The standard software (which is usually provided

\footnotetext{
${ }^{2}$ Each slice is presented as a two dimensional image which is built as a matrix (usually with fairly high resolution pxq, where $\mathrm{p}$ and $\mathrm{q}$ are a few hundred). Moreover, very frequently, for individual imaging studies, only a fraction of the gathered information is used.
}

by the vendor together with the dicom files of the study) allows for the display of the planar slices as shown in Figs. 2 and 4.

Each. $\mathrm{dcm}$ file has a header with patient information such as age, gender, height, the scanner characteristics and scanning parameters. The header also contains the geometry information that allows for the precise positioning of the data points in $3 D$ and their gray level. The corresponding fields are ImagePositionPatient, ImageOrientationPatient and PixelSpacing ${ }^{3}$. Figure 6 illustrates the origin and the axes of the plane containing the texturized rectangle corresponding to a $\mathrm{dcm}$ file. Figures 3 and 5 illustrate the (position) of the slices of two studies.

Real organs in 3D usually do not conform to planar slices. For example the human maxillofacial bone tends to follow a parabola [5]. Recently there have been efforts to explore MRI and CT volumes along non planar slices [68]. In the next subsection we consider extracting information from $3 D$ volumes using curved slices which adapt to the organs to be inspected.

\section{Painting the curved slices within a 3D volume}

The sequence of dicom files determine a volume, usually a box ${ }^{4}$

\footnotetext{
${ }^{3}$ In Matlab the access to the header is provided by the command dicominfo. It requires the Image Processing Toolbox.

${ }^{4}$ In most cases, as illustrated in Fig. 3, the box coincides with the volume occupied by the slices in 3D. But it is not always so as is exemplified in Fig. 5.
} 

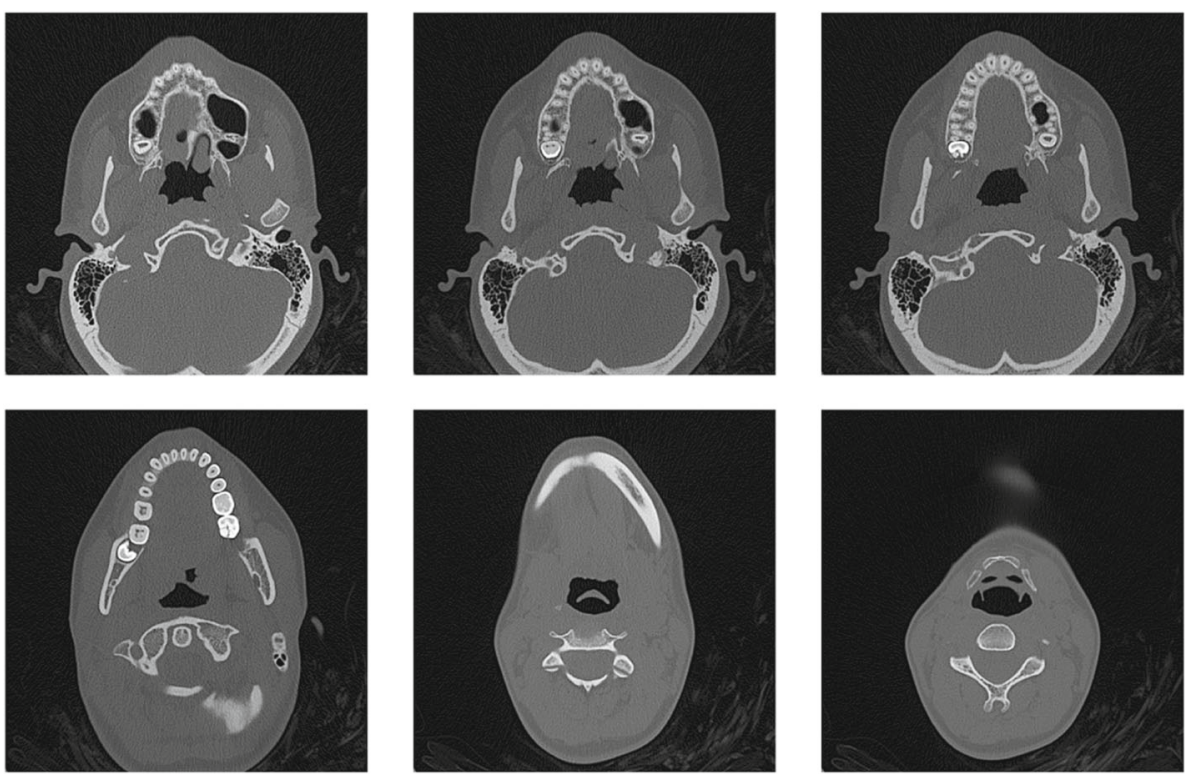

Fig. 2 Six axial dental slices, not all consecutive. Images are $512 \times 512$ pixels

$$
\left\{(x, y, z): x_{0}<x<x_{1}, y_{0}<y<y_{1}, z_{0}<z<z_{1}\right\}
$$

where $x, y$ and $z$ are the standard cartesian coordinates and $x_{i}, y_{i}$ and $z_{i}$ give the extensions of the inspected volume. The information necessary to determine these parameters is provided in the following fields of the dicom files: ImagePositionPatient, ImageOrientationPatient and PixelSpacing. The box has a natural discretization which, most of the time, may be made to coincide with the positions of the data points in the volume, as prescribed by the fields in the headers. Hence each point of the discretization may be assigned a gray level provided by the dicom file. Given a curved slice within the volume above, to each of its points we assign a gray level according to the gray levels of the nearest points in the discretization. This process is referred to as texturization and it is amply used in computer graphics to "paint" surfaces, [9].

There are various texturization techniques, two possibilities are: trilinear interpolation and nearest neighbour approximation.

\section{Trilinear interpolation}

Trilinear interpolation is a widely used technique, see $[10,11]$, in computer art and virtual reality, and in general, to display computer generated $3 \mathrm{D}$ objects. It is a mathematical algorithm that assigns a color (or a level of gray) to a point in $3 D$ space based on the colors (or levels of gray) at the vertices of the box that contains it.

More precisely, trilinear interpolation involves three linear interpolations, namely, given two points $\mathbf{a}$ and $\mathbf{b}$ in

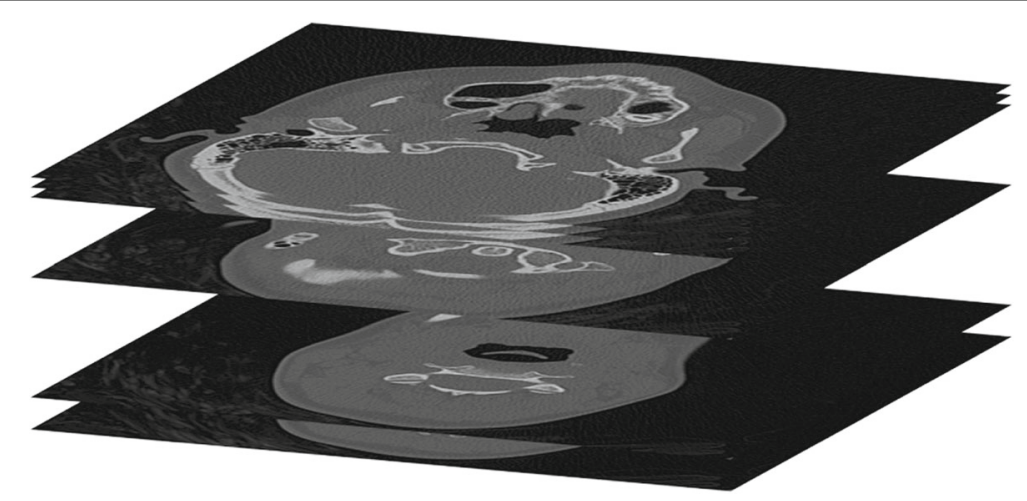

Fig. 3 Positions in space of slices of Fig. 2 

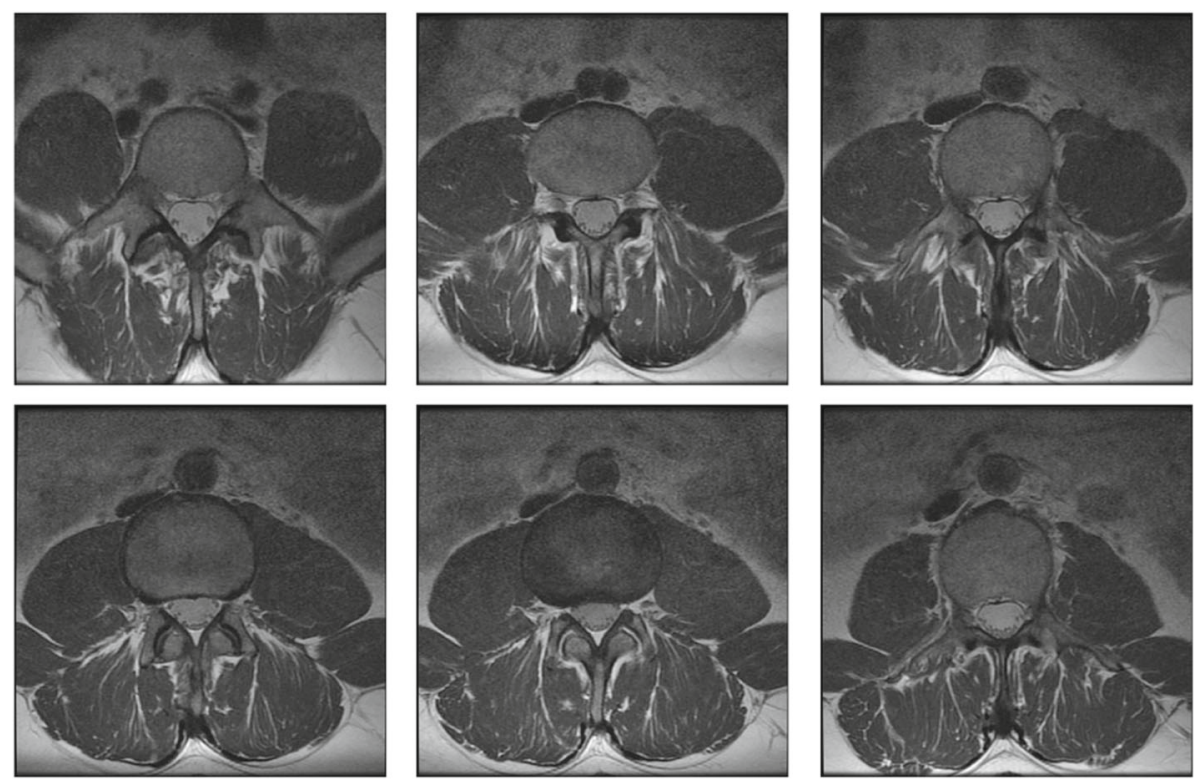

Fig. 4 Six not parallel slices of the stomach

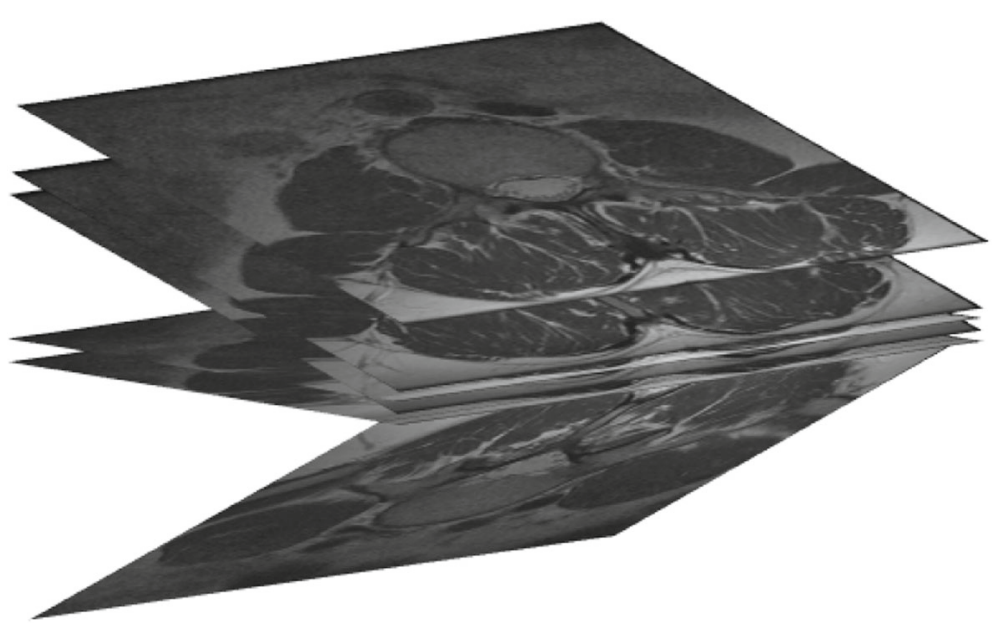

Fig. 5 Positions in space of the stomach slices of Fig. 4

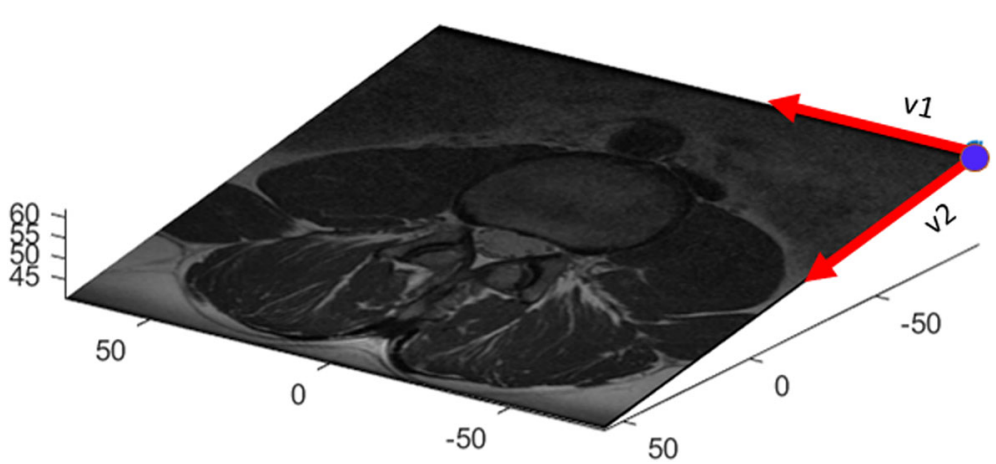

Fig. 6 Reference origin and generating vectors of the 3D plane containing a stomach slice 
$3 D$ for any point $\mathbf{x}$ on the segment joining them, there is $\lambda \in[0,1]$ such that $\mathbf{x}=(1-\lambda) \mathbf{a}+\lambda \mathbf{b}$.

Let $\mathbf{a}_{F L}, \mathbf{a}_{F R}, \mathbf{a}_{B L}, \mathbf{a}_{B R}$ and $\mathbf{b}_{F L}, \mathbf{b}_{F R}, \mathbf{b}_{B L}, \mathbf{b}_{B R}$ be the vertices of a box ${ }^{5}$, then any $\mathbf{x}$ inside the box may be written

$$
\begin{aligned}
\mathbf{x}= & (1-\alpha)(1-\beta)\left[(1-\gamma) \mathbf{a}_{F L}+\gamma \mathbf{a}_{F R}\right] \\
& +(1-\alpha) \beta\left[(1-\gamma) \mathbf{a}_{B L}+\gamma \mathbf{a}_{B R}\right] \\
& +\alpha(1-\beta)\left[(1-\gamma) \mathbf{b}_{F L}+\gamma \mathbf{b}_{F R}\right] \\
& +\alpha \beta\left[(1-\gamma) \mathbf{b}_{B L}+\gamma \mathbf{b}_{B R}\right]
\end{aligned}
$$

for some $\alpha, \beta$ and $\gamma$ in $[0,1]$.

In the case that the medical volume is built from dicom files corresponding to rectangles positioned on parallel planes in $3 D$, arranged in a box, each rectangle is discretized according to the dicom headers ${ }^{6}$ and the $3 D$ positions of these discrete points partition the volume into sub-boxes. So, any point inside the volume, but otherwise of arbitrary coordinates, is contained in one and only one sub-box and hence it may expressed as in Eq. 1 for some $\alpha, \beta$ and $\gamma$. The gray level assigned to $\mathbf{x}$ is given by Eq. 1 with the vertices substituted by their corresponding gray levels.

This process is used to assign a gray level to each point of the curved slice, and hence, for its painting.

\section{Nearest neighbor texturization technique}

Another painting technique, which takes more fully into account the gray level in the vecinity of a point in the curvilinear slice, as opposed to interpolating it from near points is referred to as the nearest neighbor approximation. We will discuss a slightly modified version of it which takes into account a prescribed discretization of the curved slice. It consists of the following steps:

- Choose a discretization of the curved slice given by the parametric surface, i.e. the map

$[0,1] \times[0,1] \rightarrow S$, where $S$ fits the object of interest. In Fig. $7 S$ is the half ellipsoid fitting the rotator cuff muscle complex. The position of $S$ is informed by the humerus head (green points). The discretization of $S$ is induced by the discretization of $[0,1] \times[0,1]$ via the above map, and we have to assign a gray level to each point of the discretization of $S$.

- Partition the volume bounding the set of slices in $3 D$ into sub-boxes. This subdivision does not rely upon the orderly distribution of the data points in the volume, on it conforming to be a box or on the slices to be parallel.

\footnotetext{
${ }^{5}$ In $\mathbf{a}_{F L} F$ and $L$ refer to front and lower (similarly $B$ and $R$ ), the a vertices lie in one face and the $\mathbf{b}$ vertices correspond to its parallel face.

${ }^{6}$ As stated above, the information regarding the positions of the planes and the positions of the points may be computed from the information in the fields ImagePositionPatient, ImageOrientationPatient and PixelSpacing in the headers.
}

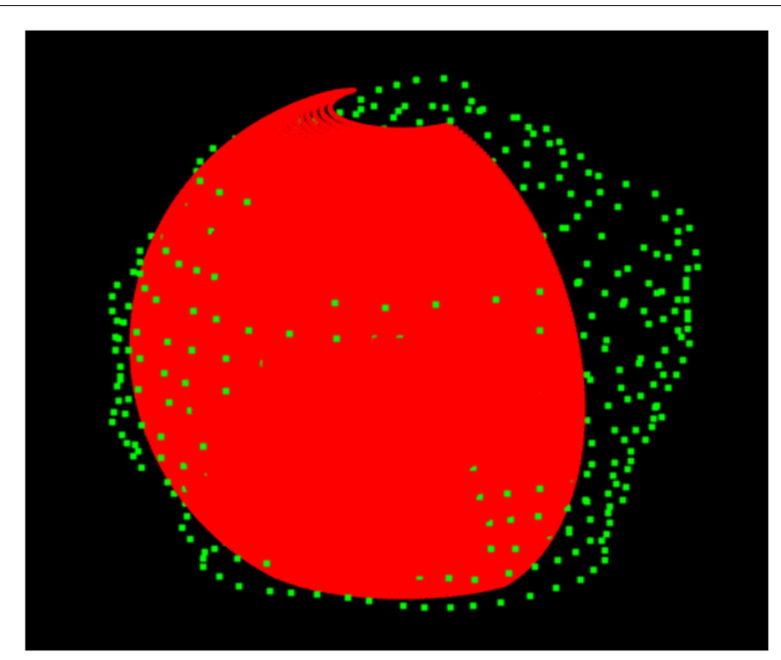

Fig. 7 Green: Points on the humerus head surface - Red: Fitting ellipsoid

- Each point of the curved slice's discretization belongs to some sub-box so we assign to it the gray level of the nearest data point of that sub-box ${ }^{7}$.

Each one of these two techniques, has particular advantages regarding the information that is retrieved along the curved slice. Trilinear interpolation tends to produce smoother displays and nearest neighbor approximation is very faithful in terms of the gray level assigned to each point since no smoothing is applied. See Fig. 8 for a comparison of a curved slice painted with trilinear interpolation and the nearest neighbor method.

\section{Results}

We consider three visualization examples along curvilinear surfaces fitted to objects of interest:

- the humerus in order to detect tears in the rotator cuff muscle complex

- the jaw to view a curvilinear section to provide a panoramic view of all dental pieces at the same time

- the aorta to check aortic aneurysm status

\section{MRI: Detecting tears near the humerus head}

The muscle and tendon complex surrounding the humerus head is prone to sport injuries and age related degenerations, which usually show up as tears. Shoulder MRI is a standard procedure to resolve the position and extent of the tear and it guides the clinician regarding the choice of treatment.

Figure 9 illustrates the texturization of the fitting ellipsoid in Fig. 7 assigning gray levels to the points of its

\footnotetext{
${ }^{7}$ It may happen that some sub-boxes do not contain data points, so for a point in the discretization of the curved slice which lies in this sub-box no gray level is assigned.
} 

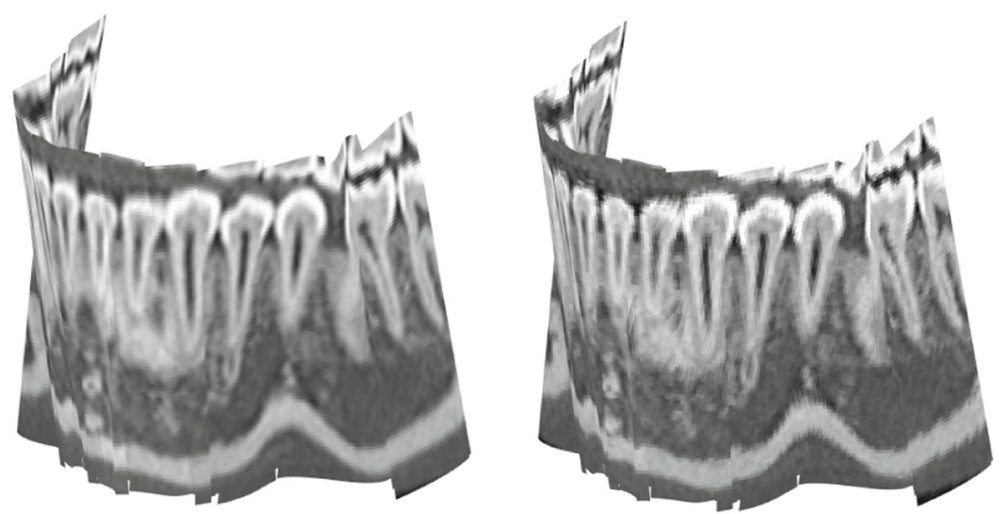

Fig. 8 Left: Curved slice texturized by trilinear interpolation using $511 \times 511 \times 165=43.084 .965$ data points. Right: Same curved slice texturized by nearest neighbor using $20 \times 20 \times 20=8.000$ sub-boxes

discretization. It uses the nearest neighbor technique presented in the previous section, whose salient feature is the possibility to choose the number of sub-boxes. In fact, as the number of sub-boxes increases the number of empty boxes and hence the number of uninformed points of the ellipsoid discretization also grows. Figure 9 depicts the texturization for $17^{3}=4.913$ sub-boxes and Fig. 10 shows the same ellipsoid's texturization when the number of sub-boxes have been increased to $20^{3}$.

The two renderings are complementary, the $17^{3}$ subbox rendering shows the approximate position of the tear (in our case infraespinatus and teres minor) on the fitting ellipsoid and the $20^{3}$ sub-box rendering pinpoints more precisely the points for which there is no dicom information.

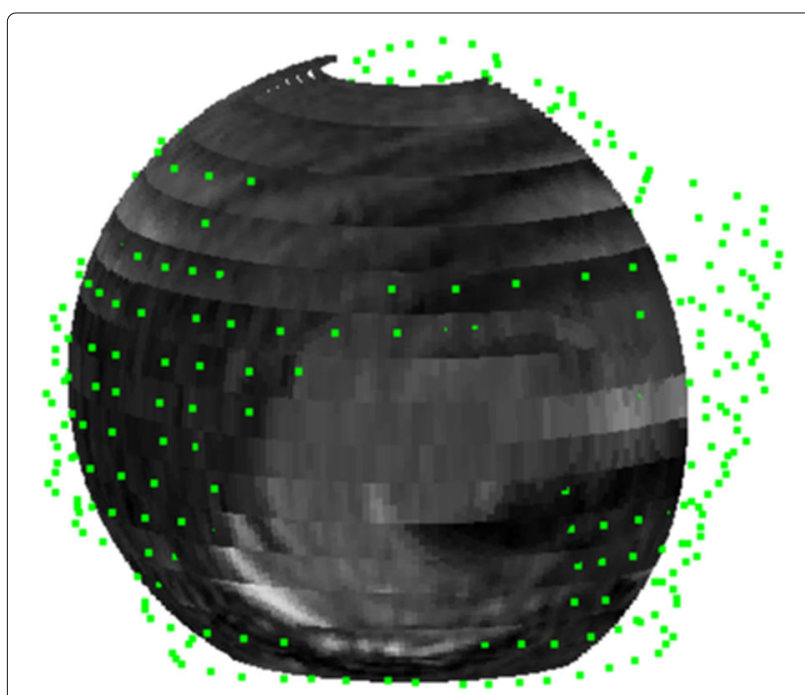

Fig. 9 Curved slice texturized by nearest neighbor using $17^{3}=4.913$ sub-boxes
Additionally to the tear information on the fitting ellipsoid we obtain shape and size information of the tears by considering further surfaces of revolution, namely by offsetting the circles composing the fitting ellipsoid's circles ${ }^{8}$.

The MRI volume illustrated in Fig. 11 shows three curved slices, the middle one is half of an ellipsoid of revolution fitting the humerus head ${ }^{9}$ and two additional curved slices, one inward (radially towards the ellipsoid axis, i.e. towards the humerus bone) and the other outwards, away from the axis. Each curved slice has beeen painted with the nearest neighbor method using sixteen MRI slices and $17^{3}=4.913$ sub-boxes. The radial distance between the inner and the outermost curved slices in $3 D$ is $10 \mathrm{~mm}$. They illustrate the infraespinatus and teres minor area which show bright zones suggesting tears. Figure 12 illustrates the $3 D$ rendering of the tears with the humerus head described by green points. The web version of this paper offers animations of $3 D$ renderings of tears of the rotator cuff complex based on the fitting ellipsoid and several outer and inner slices. The latter are surfaces of revolution obtained by augmenting the radii of the ellipsoid circles in the normal direction to its axis.

\section{$\mathrm{CT}$ : Curved slices in dentistry}

In dental applications, $3 D$ visualization of individual or groups of teeth has been of increasing interest since the introduction of cone beam computed tomography $(\mathrm{CBCT})$. This is specially the case in dentistry in the areas of implantology and endodontics. In implantology The American Academy of Oral and Maxillofacial Radiology, recommends CBCT for presurgical assessment of dental implant sites. In the field of endodontics in the

\footnotetext{
${ }^{8}$ The fitting ellipsoid consists of circles that lie on parallel planes and their centers live on an orthogonal line to these planes. An offset surface consists of circles with the same centers and their radii are increased or decreased by a small fixed number.

${ }^{9}$ The fitted ellipsoid mostly contains the humerus bone head.
} 


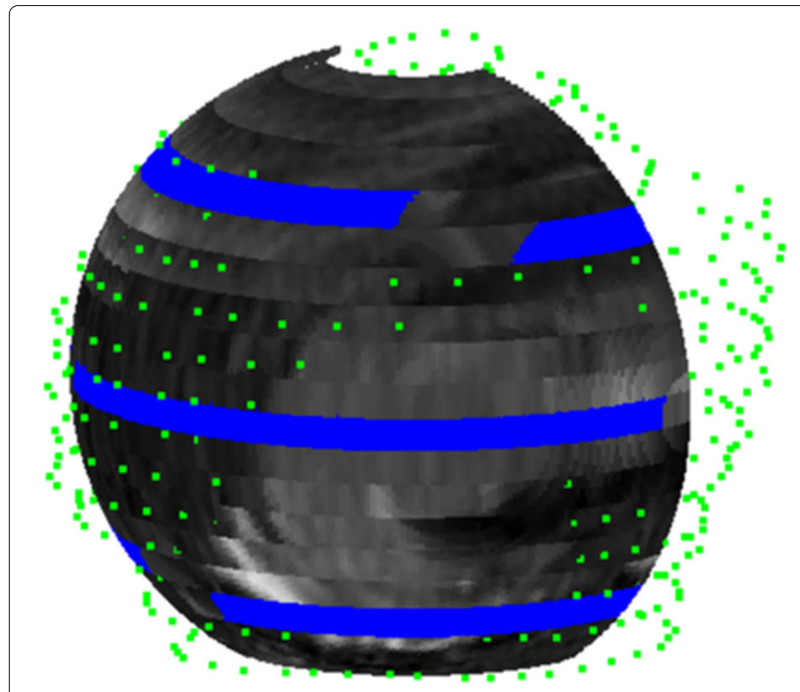

Fig. 10 Blue areas of the curved slice correspond to points which lie in sub-boxes for which there is not DICOM provided information

recent book, The Guidebook to Molar Endodontics [12] the authors argue the convenience of CBCT, particularly in establishing the morphology of the area of the root system, namely the existence and $3 D$ placement of isthmi and intracanal communication in molars ${ }^{10}$. Moreover, the utility of the knowledge of the surrounding tissues, by inspection with $\mathrm{CBCT}$, has been subject of recent interest because, potentially, it has a better sensitivity and specificity than intraoral periapical radiography variations, see [13].

In this subsection we consider special types of curved slices that adapt to morphologies of interest in maxillofacial and dental imaging. Figure 8 illustrates a curved slice extracted from a tomographic volume of a human jaw. The slice follows the dental pieces allowing for their full display, together with roots, in $3 D$. Due to maxillofacial morphology the most useful types of curved slices are given by ruled surfaces. These are composed of straight line segments. A specially interesting subfamily of ruled surfaces are the developable surfaces which enjoy the property that may be flattened without metric distortion, which means that lengths and areas are exactly the same as measured on the curved slice or its flat display ${ }^{11}$.

Construction and texturization of curved slices described by ruled surface has been considered in [6] mainly in the case of cylinders. For another proposal for the flattened presentation of texture information along a curved slice see [14]. In odontology, [15] considered

\footnotetext{
${ }^{10}$ The book contains engaging illustrations of various types of root canal systems which are usually hard to treat.

${ }^{11}$ Mathematically, the 1:1 mapping between a developable surface and its flat image is referred to as an isometry.
}

developable surfaces along Bézier curves and [8], cone splines built with circular cones.

Developable surfaces are usually built along $3 D$ curves and in maxillofacial applications follow the sequence of dental pieces. There are various ways to build a developable surface that contains a prescribed curve, see [16] for a reference in the more theoretical context of Computer Aided Geometric Design and [8] for $G^{1}$ conic splines built ${ }^{12}$ along an approximating $G^{1}$ curve consisting of circle segments. The latter uses very beautiful classical geometry ideas due to Fuhs and Stachel [17].

A simplification occurs when the curve along which the developable surface has been built gets mapped into a straight line in the flattened image as illustrated in Fig. 13.

A potentially useful application is the display of the texturized developable surface containing a root canal as displayed in Fig. 14.

Figure 15 shows how the above texturized surface is built as a ruled surface, i.e. composed from straight line segments, and the flat (and texturized) image is presented in Fig. 16. In this particular example the $3 D$ curve that approximates the root canal is mapped to a straight line segment.

Figure 17 shows two developable surfaces along the root canal allowing for the inspection of the neighboring tissues along two surfaces that intersect transversally along a canal root approximating curve.

For more information on the usefulnes of imaging in root canal treatment see [18-20].

\section{CT: Aortic aneurysms}

An aortic aneurysm is a localized dilation of the aorta. Aneurysms rank among the leading causes of mortality in the United States. They might be asymptomatic in which case their growth should be monitored because of even higher mortality rates if rupture occurs, [21]. A recent review is [22].

CT scans can be used to provide information about the $3 D$ geometric shape of the aneurysms by looking at its curved sections along the centerline of the aorta. Figure 18, shows two mathematical surfaces that contain the center curve of an aneurysm, and are positioned transversally with respect to each other in $3 D$. In the web version of the paper the surfaces are black and magenta, the cloud of blue points tracks the surface of the aorta, the red points sample the curve of centers of the aneurysm and the yellow line is its degree eight Bézier curve approximation. The technique utilized to create the developable surface is due to Aumann, see [16].

Figure 19 shows both texturized surfaces, together with the $3 D$ point cloud representing the surface of the aneurysm and Fig. 20 their isometrically flattened versions.

\footnotetext{
${ }^{12} G^{1}$ refers to geometrically continuous joining of curves or surfaces.
} 

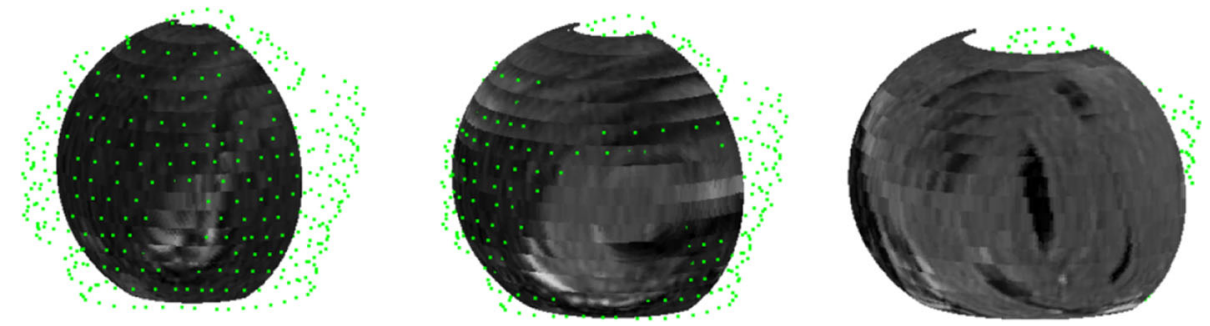

Fig. 11 Middle: Ellipsoid of revolution fitting the humerus head. Left: Inward 4mm. Right: Outward $6 \mathrm{~mm}$

\section{Mathematical techniques}

We use various types surfaces in the examples presented in this paper. In the case of aneurysms and the human jaw the parametric surface that represents a curved slice within a medical is chosen within the family of developable surfaces. These surfaces may be flattened without distortion, namely the lengths, shapes and angles are the same as measured along the $3 D$ surfaces and in their flat versions. The simplest examples of developable surfaces are cones and cylinders ${ }^{13}$.

We use two ways to build developable surfaces:

- In case of the curved slice of the jaw we use cone splines. These are built stiching together sections of cones, along common generators, such that any pair of adjacent cones share the same tangent plane along their common generator.

- For curved slices along an aneurysm we build a developable surface along a Bézier curve approximating the centerline of the aorta using Aumann's algorithm [16]. Automatic detection of the aorta centerline has been recently discussed in [23] and the approximating Bézier curve is calculated using least squares.

To flatten without distortion a developable surface, we need to construct an explicit isometry between the surface and an open connected set in the plane, see [24]. A more efficient method, that works for any type of developable surface and does not require finding the explicit isometry, is based on the fact that the geodesic curvature of a curve on the surface does not change after isometric unfolding the surface into the plane. Namely, let $\alpha$ be a curve on the surface $S$ and denote by $\bar{\alpha}$ its image under isometric unfolding of $S$. If $\kappa_{g}$ is the geodesic curvature of the curve $\alpha^{14}$ on the surface $S$ and $\bar{\kappa}^{15}$ is the curvature of its image under isometric unfolding then $\bar{\kappa}=\kappa_{g}$, see [25].

\footnotetext{
${ }^{13}$ In general, a developable surface is a ruled surface such that the tangent plane is the same along each ruling.

${ }^{14}$ The geodesic curvature of curve on a surface measures how much it deviates from a geodesic, i.e. a curve that minimizes distance.

${ }^{15}$ The curvature of a planar curve measures how much it deviates from a straight line.
}

The goal of the method is to construct a planar curve $\bar{\alpha}$ with curvature $\bar{\kappa}=\kappa_{g}$ and then build the unfolded surface by drawing the generators at the apprpriate angle to the planar curve $\bar{\alpha}$.

Recall the Frenet equations for the planar curve $\bar{\alpha}$,

$$
\begin{aligned}
& \bar{e}_{1}^{\prime}=\bar{\kappa} \bar{e}_{2} \\
& \bar{e}_{2}^{\prime}=-\bar{\kappa} \bar{e}_{1}
\end{aligned}
$$

where $\bar{e}_{1}^{\prime}$ and $\bar{e}_{2}^{\prime}$ are the derivatives, with respect to arc length, of the unit tangent and the normal vector of $\bar{\alpha}$, respectively.

Applying the chain rule and considering $v(t)=$ $\|\dot{\alpha}(t)\|=\|\dot{\bar{\alpha}}(t)\|$ we get:

$$
\begin{aligned}
& \dot{\bar{e}}_{1}(t)=v(t) \kappa_{g}(t) \bar{e}_{2}(t) \\
& \dot{\bar{e}}_{2}(t)=-v(t) \kappa_{g}(t) \bar{e}_{1}(t)
\end{aligned}
$$

Since $\bar{e}_{1}$ and $\bar{e}_{2}$ are unit vectors then let $\bar{e}_{1}=$ $[\cos (\phi(t)), \sin (\phi(t))]$ for an unknown real value function $\phi$. Thus $\phi^{\prime}(t) \bar{e}_{2}(t)=\dot{\bar{e}}_{1}(t)=v(t) \kappa_{g}(t) \bar{e}_{2}(t)$. So

$$
\phi(t)=\int v(t) \kappa_{g}(t) d t
$$

Since $\bar{e}_{1}(t)$ is a unit vector, then $\dot{\bar{\alpha}}(t)=v(t) \bar{e}_{1}(t)$. Therefore the family solution of congruent curves is given by:

$$
\bar{\alpha}(t)=\int v(t)[\cos (\phi(t)), \sin (\phi(t))] d t
$$

After the construction of the planar curve $\bar{\alpha}$ the final step is to construct the rulings of the unfolded surface preserving the angle enclosed by the tangent vector and the generator in $3 D$. See Fig. 13 for an illustration of this method where the red curve $\alpha$ on the surface is a geodesic, then $\kappa_{g}=0$ and hence the curve $\bar{\alpha}$ is a straight line. Additional file 4 is a video of the 3D setup of the flattening method. Figure 14 shows a dental application where the magenta curve is a geodesic which interpolates the canal root of the tooth, this is why the canal root looks as a straight line in Fig. 16. 

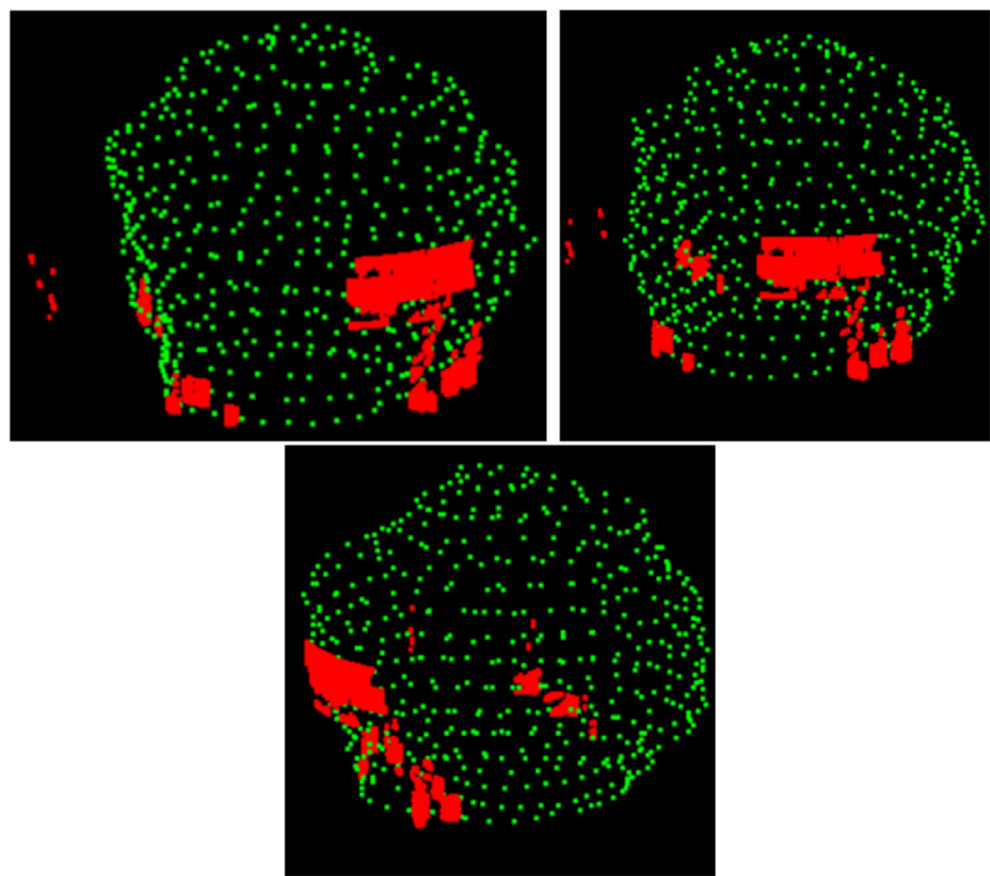

Fig. 12 Three views of the humerus head and the tears. Green: Humerus head points. Red: Tears, Additional file 5

\section{Discussion}

We propose elementary differential geometry and texturization methods to inspect $3 D$ medical volumes arising from MRI and CT studies. The method is based on fitting surfaces to the object of interest and common computer graphics texturization techniques.

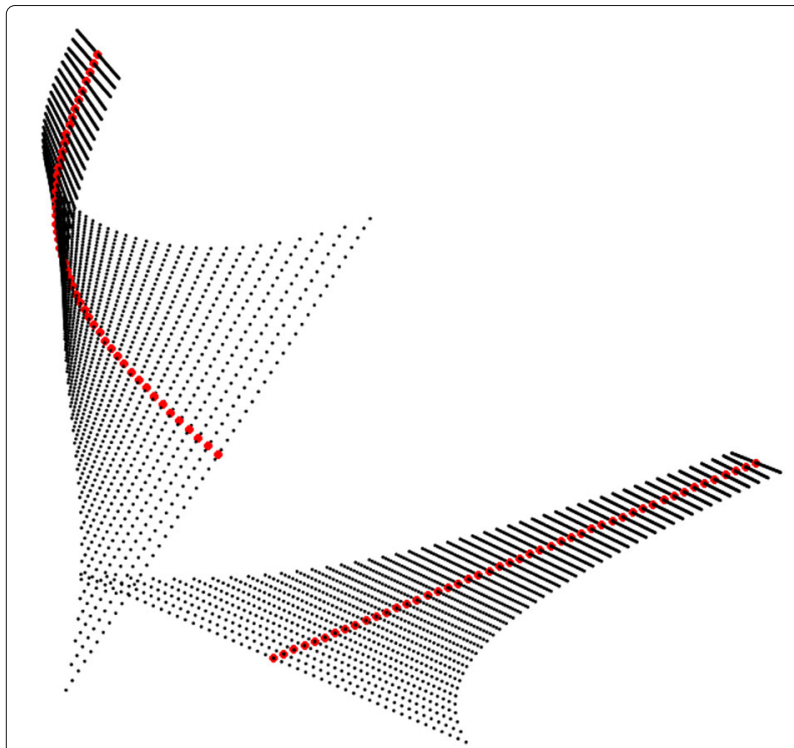

Fig. 13 Unfolding of a developable surface along a pregeodesic (red) and its planar display, Additional file 3
We consider three examples

- rotator cuff tear visualization,

- abdominal aortic aneurysm display along transversal curved slices and

- odontological visualization.

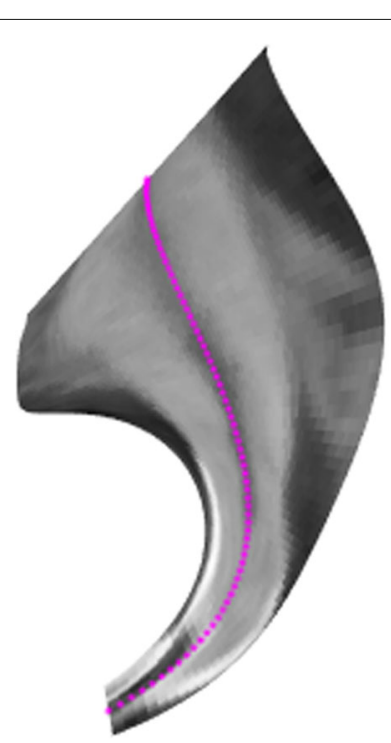

Fig. 14 Developable surface along a space Bézier cubic that follows the root canal. We provide a video that demonstrates graphically the $3 D$ shape of this developable surface in the Additional file 1 in the website version of this paper 


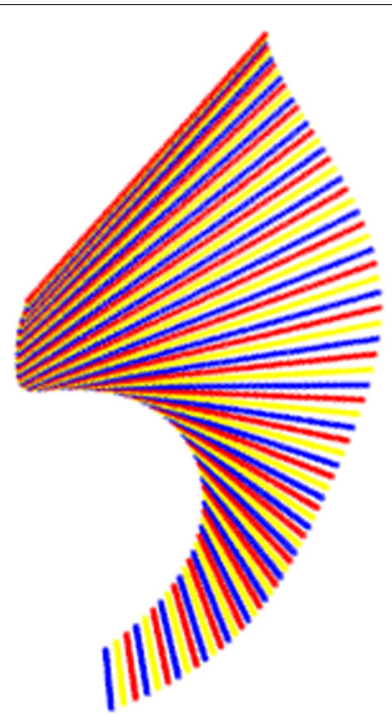

Fig. 15 Developable surface as composed of line segments

The examples pertain to very different fields of medical healthcare which, from the visualization perspective, are treated in a fairly unified way, using parametric surfaces placed within a medical volume. The techniques are applied to the most common medical volumes: MRI and CT, but in principle may be applied to any volumes, gathered with any methodology. The visualization technique is based on two elements: choosing the surface in the area of interest which entails a fitting process and the texturization of this surface. The data points of the volume do not have to be organized in any structured way. The nearest neighbor technique delivers faithful texturizations of parametric surfaces for any reasonable data point distributions.

The technique can be extended to volumetric inspection by moving the texturized surface within the medical volume. This proves useful to produce animations (see the rotator cuff animation in the Additional file 1 of the web version of this paper). The technique also

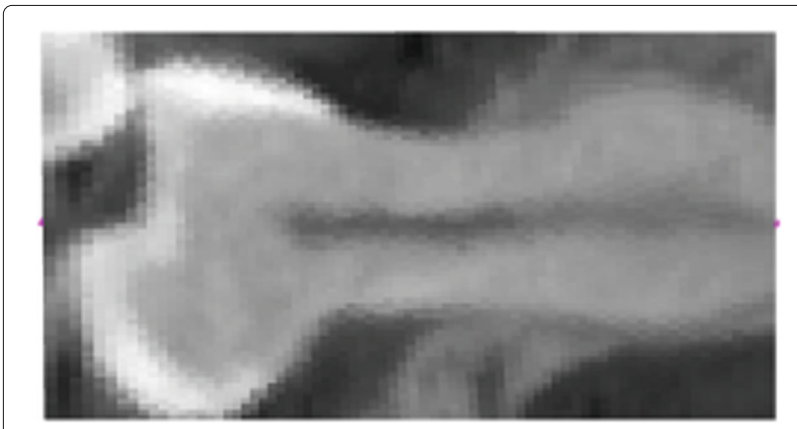

Fig. 16 Flattened developable surface corresponding to Fig. 14

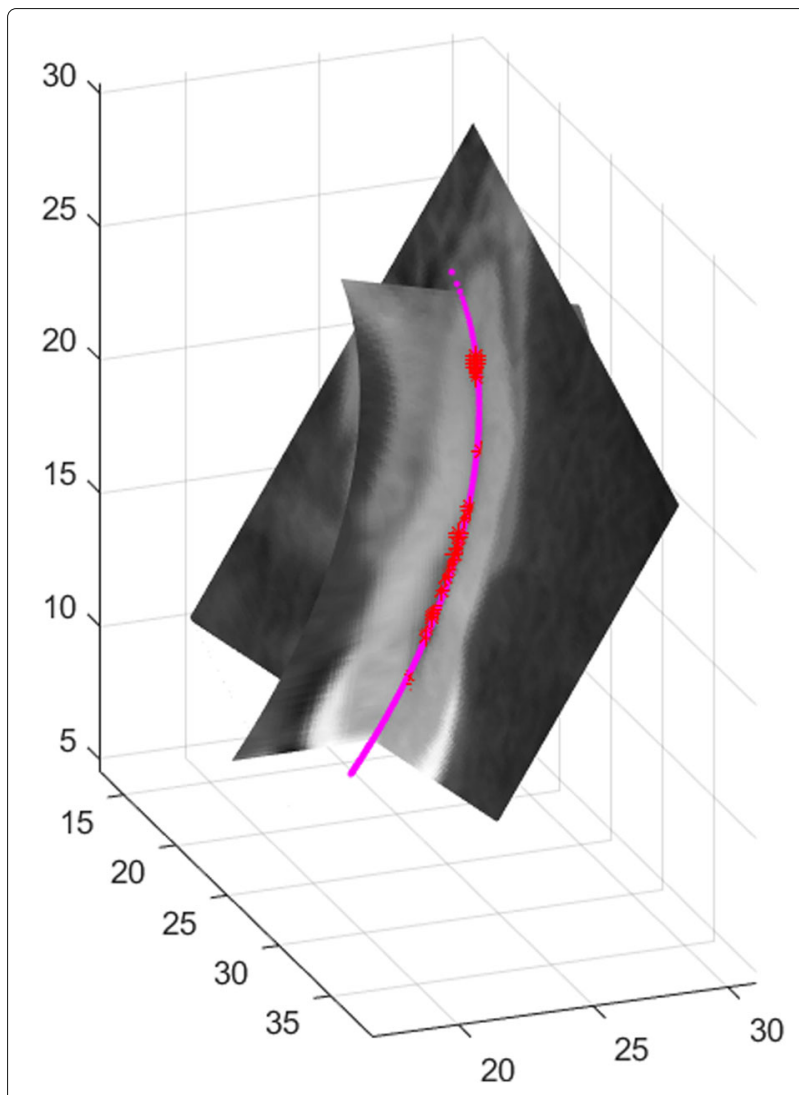

Fig. 17 Two transversal surfaces along the root canal

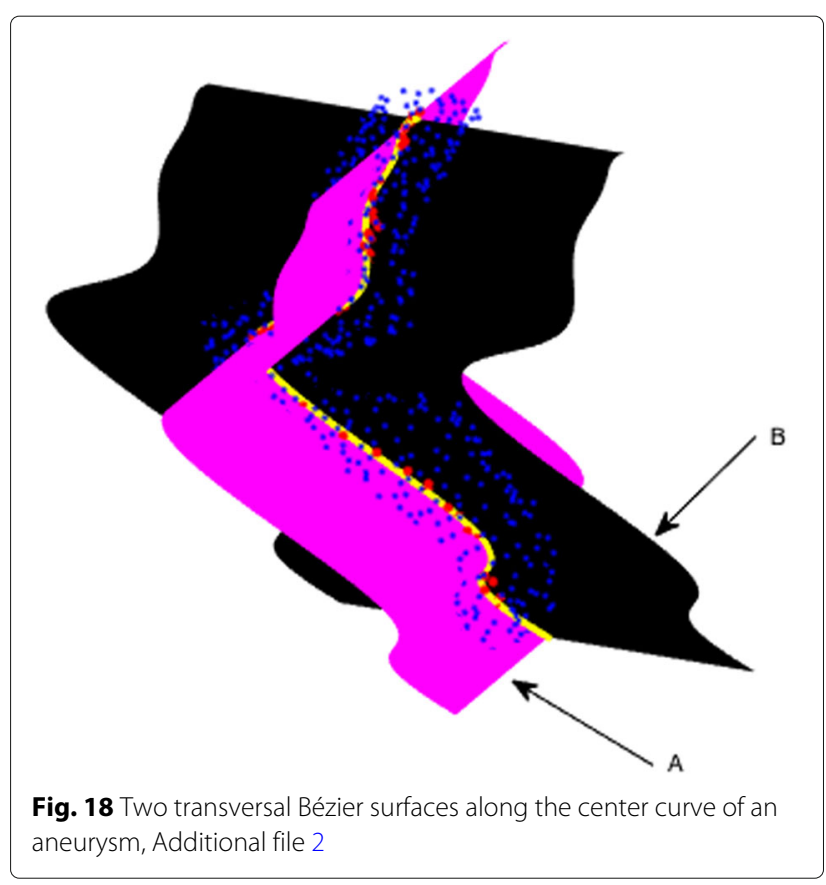




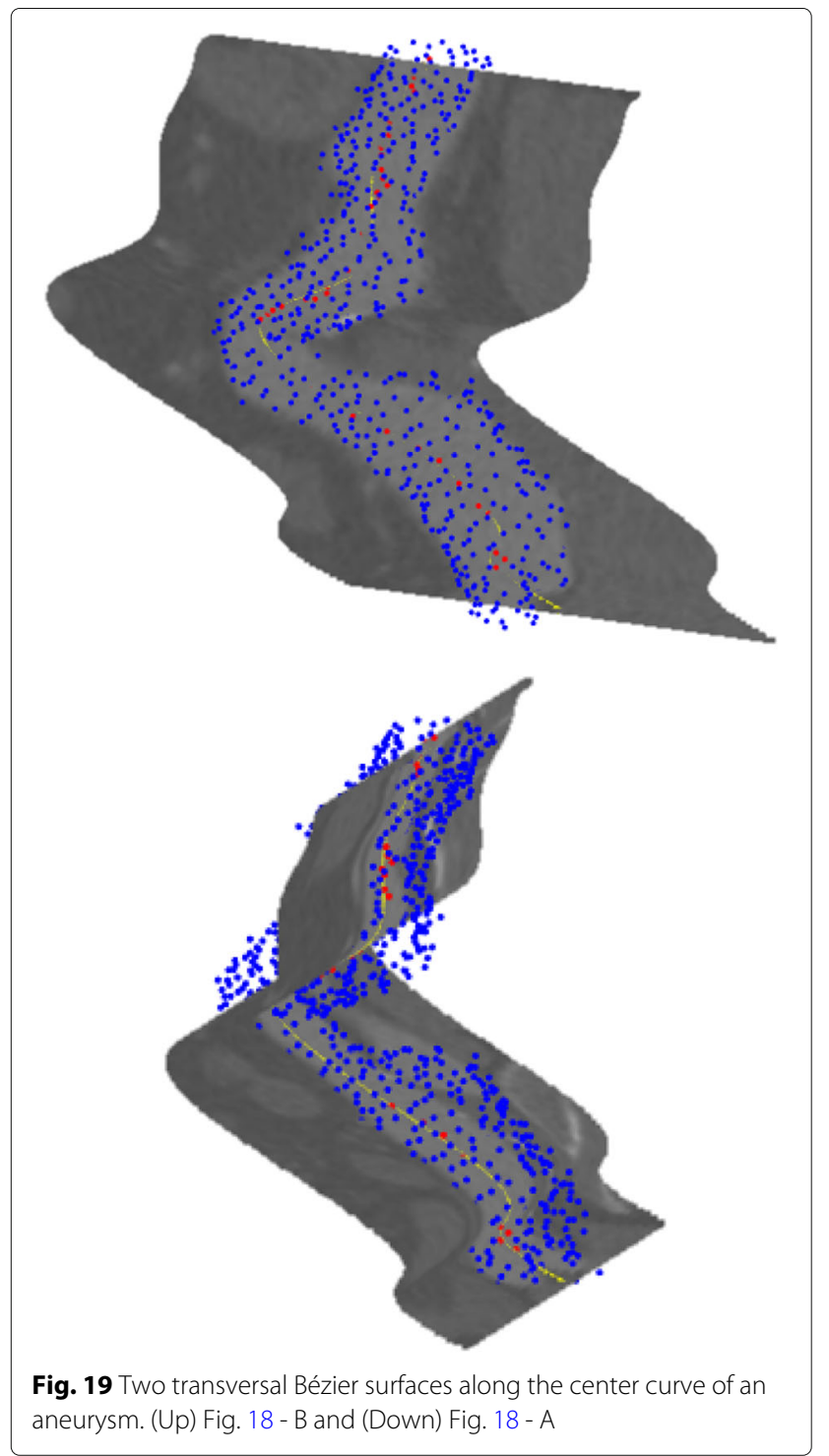

offers visualization of the morphology along the intersection of transversal surfaces, as illustrated in the case of aortic abdominal aneurysms and along the root canal of a dental piece. In the case of aortic aneurysms and the jaw, the curvilinear surfaces may be chosen to be developable, which allows for their flat display without deformation.

In the case of the rotator cuff we illustrate the technique by taking a sequence of offsets, i.e. of "parallel" fitting surfaces which allows for the localization of possible tears within the $3 D$ volume. The research shows that it is possible to offer high quality visualization without the need of specialized software.

It is not difficult to envision other applications in medical visualization such as vascular calcifications and implantology in dentistry, among others.

Finally we would like to bring forward the idea that texturized curved slices can be used as navigation devices within medical volumes. Moreover, since are given by parametric surfaces their shape can be changed as well as their position. In the particular case that the surface is developable it may be flattened without distortion.

The main advantage of texturized curved slices is lower cost as compared volumetric rendering of organs, flexibility (shape change) and adaptability (position change).

\section{Conclusions}

The inspection of medical volumes given by the texturization of surfaces adapted to the geometry of the object of interest is possible using surface techniques of differential geometry and texturization processes of common use in computer graphics. The technique was illustrated in various MRI and CT examples, including the inspection of rotator cuff injuries, aortic aneurysms and in dental visualization. Since the technique is relatively computationally inexpensive it should play a role in the clinical practice, especially in low to medium income communities. More
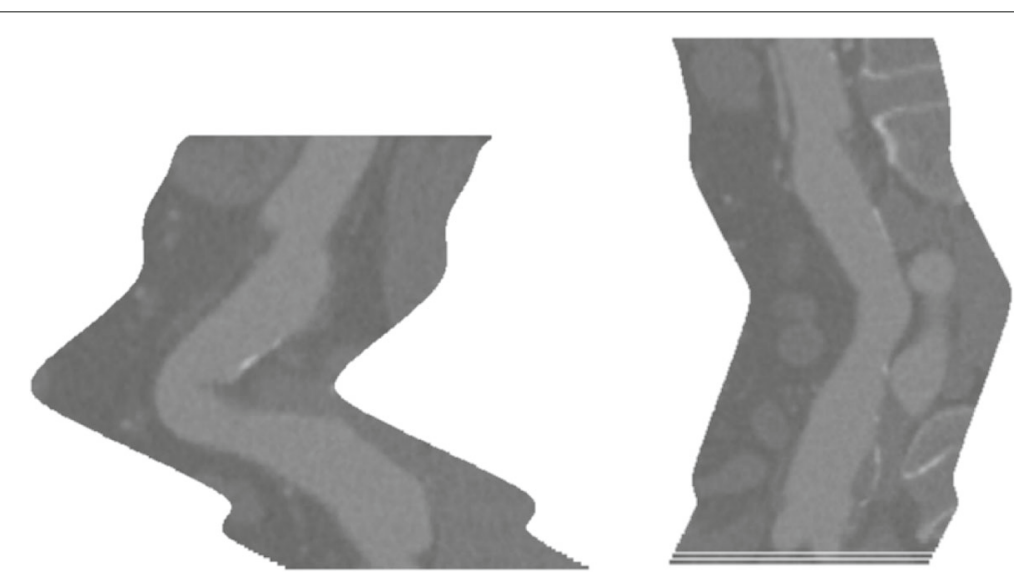

Fig. 20 Isometrically flattened versions of the last two developable surfaces 
interdisciplinary efforts will be necessary to bring such a possibility to fruition.

\section{Supplementary information}

Supplementary information accompanies this paper at https://doi.org/10.1186/s12911-019-1018-2.

Additional file 1: Dental: Developable surface containing the root canal as geodesic.

Additional file 2: Aortic aneurysm: Two transversal developable surfaces containing the centerline of an abdominal aortic aneurysm.

Additional file 3: Mathematical illustration of the planar unfolding of a developable surface. Its geodesic curve maps into a straight line segment. Sizes and shapes are preserved.

Additional file 4: Illustration of the isometric unfolding process. It flattens a developable surface without stretching distortion.

Additional file 5: Rotating cuff: Positioning of tears with respect to the humerus head.

\section{Abbreviations}

$\mathrm{CT}$ : computed tomography; DICOM: Digital imaging and communications in medicine; MRI: magnetic resonance imaging

\section{Acknowledgments}

Both authors acknowledge the financial support of Colciencias, through project GeoMed and M. Paluszny acknowledges the support of Universidad Nacional de Colombia. Both authors thank the reviewers for observations that allowed us to improve the paper.

\section{Authors' contributions}

MP designed the methodology to build the new visualization techniques to display medical information on curved surfaces together with their flat display in the case of aneurysms and odontological data. DR developed and wrote the Matlab scripts. Both authors contributed equally to the final writeup of the paper. Both authors read and approved the final manuscript.

\section{Funding}

The research was funded by Colciencias through the GeoMed project and our Alma Mater institution: Universidad Nacional de Colombia (UNAL), Sede Medellín. Colciencias provided financial support for Dany Ríos work and UNAL paid the salary of Marco Paluszny and covered partially the processing costs of the datasets used in the research. The main goals of the research were defined through the GeoMed project. The actual choice of examples presented in this work is the responsability of M. Paluszny.

\section{Availability of data and materials}

The sources of the datasets used in this study are as follows: the abdominal aortic aneurysm data were pubicly available in the world wide web, the rotator cuff and the odontological datasets are from the personal library of $\mathrm{M}$. Paluszny. The datasets are available from the authors upon request.

\section{Ethics approval and consent to participate}

Not applicable

\section{Consent for publication}

Not applicable.

\section{Competing interests}

The authors declare that they have no competing interests.

Received: 3 September 2019 Accepted: 30 December 2019

Published online: 07 February 2020

\section{References}

1. Fuchs $H$, Levoy M, Pizer S (1989) Interactive visualization of $3 d$ medical data. Computer 22(8):46-51

2. Zhang Q, Eagleson R, Peters TM (2011) Volume visualization: a technical overview with focus on medical applications. J Digit Imaging 24(4):640-664
3. Wheeler G, Deng S, Toussaint N, Pushparajah K, Schnabel J, Simpson J, Gomez A (2018) Virtual interaction and visualization of $3 d$ medical imaging data with VTK and Unity. Health Care Technol Lett 5(5):148-153

4. Smith-Bindman R, Miglioretti DL, Johnson E, Lee C, Feigelson HS, Flynn M, Greenlee RT, Kruger RL, Hornbrook MC, Roblin D (2012) Use of diagnostic imaging studies and associated radiation exposure for patients enrolled in large integrated health care systems, 1996-2010. Jama 307(22):2400-2409

5. Preti G, Pera P, Bassi F (1986) Prediction of the shape and size of the maxillary anterior arch in edentulous patients. J Oral Rehabil 13(2):115-125

6. Figueiredo $\mathrm{O}$, Hersch RD (2002) Parallel unfolding and visualization of curved surfaces extracted from large 3D volumes. J Electron Imaging 11(4):423-434

7. González C, Pérez MI, Lentini M, Ríos D, Albrecht G, Paluszny M (2017) Dental information along curved slices. In: Computing Conference. IEEE. pp 1410-1413. https://doi.org/10.1109/sai.2017.8252279

8. González C, Paluszny M (2015) Odontological information along cone splines. In: Analysis, Modelling, Optimization, and Numerical Techniques. Springer, Berlin. pp 219-233

9. Gibbs J, Petty DD, Robins N, et al. (2002) Painting and rendering textures on unparameterized models. In: ACM Transactions on Graphics (TOG), vol. 21. ACM. pp 763-768. https://doi.org/10.1145/566570.566649

10. Buss SR (2003) 3-D Computer Graphics: a Mathematical Introduction with OpenGL. Cambridge University Press, Cambridge

11. Bourke P (1999) Trilinear Interpolation. http://paulbourke.net/ miscellaneous/interpolation/. Accessed 19 Jan 2020

12. Peters OA (2016) The Guidebook to Molar Endodontics. Springer, Berlin

13. Petersson A, Axelsson S, Davidson T, Frisk F, Hakeberg M, Kvist T, Norlund A, Mejàre I, Portenier I, Sandberg H (2012) Radiological diagnosis of periapical bone tissue lesions in endodontics: a systematic review. Int Endod J 45(9):783-801

14. Vrtovec T, Likar B, Pernus F (2005) Curved planar reformation of CT spine data. In: Medical Imaging 2005: Image Processing, vol. 5747. International Society for Optics and Photonics. pp 1446-1457. https://doi.org/10.1117/ 12.595113

15. Paluszny M (2011) Between developable surfaces and circular cone splines: curved slices of 3D volumes. In: Medical Imaging 2011: Visualization, Image-Guided Procedures, and Modeling, vol. 7964. International Society for Optics and Photonics. p 796435. https://doi.org/ 10.1117/12.872157. Accessed 19 Jan 2020

16. Aumann G (2003) A simple algorithm for designing developable Bézier surfaces. Comput Aided Geom Des 20(8-9):601-619

17. Fuhs W, Stachel H (1988) Circular pipe-connections. Comput Graph 12(1):53-57

18. Paqué F, Balmer M, Attin T, Peters OA (2010) Preparation of oval-shaped root canals in mandibular molars using nickel-titanium rotary instruments: a micro-computed tomography study. Journal of Endodontics 36(4):703-707

19. Peters $O A$, Paqué $F$ (2011) Root canal preparation of maxillary molars with the self-adjusting file: a micro-computed tomography study. J Endod 37(1):53-57

20. Karabucak B, Bunes A, Chehoud C, Kohli MR, Setzer F (2016) Prevalence of apical periodontitis in endodontically treated premolars and molars with untreated canal: a cone-beam computed tomography study. J Endod 42(4):538-541

21. Woo J, Greene C Management of thoracic aortic aneurysm in adults. https://www.uptodate.com/contents/management-of-thoracic-aorticaneurysm-in-adults. Accessed 19 Jan 2020

22. Saliba E, Sia Y, Dore A, El Hamamsy I (2015) The ascending aortic aneurysm: When to intervene? IJC Heart Vasculature 6:91-100

23. Alam F, Rahman SU, Rahman AU (2018) An algorithm for extracting centerline of the aorta from CT/MR 3D images. In: 2018 6th International Conference on Biological and Medical Sciences (ICBMS 2018). Seoul National University. pp 176-180. https://dl.acm.org/doi/10.1145/3278229. 3278234. Accessed 19 Jan 2020

24. Do Carmo MP (1976) Differential Geometry of Curves and Surfaces. Prentice Hall, Englewood Cliffs

25. Pottmann H, Wallner J (2009) Computational Line Geometry. Springer, Berlin

\section{Publisher's Note}

Springer Nature remains neutral with regard to jurisdictional claims in published maps and institutional affiliations. 